\title{
Pallidotomy after chronic deep brain stimulation
}

\author{
Kristian J. Bulluss, F.R.A.C.S., M.B.B.S., Ph.D., Erlick A. Pereira, D.M., \\ Carole Joint, Ph.D., R.N., And Tipu Z. Aziz, M.D. \\ Oxford Functional Neurosurgery and Experimental Neurology Group, Nuffield Department of Surgery, \\ University of Oxford, and Department of Neurological Surgery, John Radcliffe Hospital, Oxford, \\ United Kingdom
}

\begin{abstract}
Recent publications have demonstrated that deep brain stimulation for Parkinson's disease still exerts beneficial effects on tremor, rigidity, and bradykinesia for up to 10 years after implantation of the stimulator. However with the progression of Parkinson's disease, features such as cognitive decline or "freezing" become prominent, and the presence of an implanted and functioning deep brain stimulator can impose a profound burden of care on the clinical team and family. The authors describe their experience in treating 4 patients who underwent removal of the implanted device due to either progressive dementia requiring full-time nursing or due to infection, and who subsequently underwent a unilateral pallidotomy.

(http://thejns.org/doi/abs/10.3171/2013.8.FOCUS13293)
\end{abstract}

KEY Words $\bullet \quad$ subthalamic nucleus $\bullet \quad$ globus pallidus internus
deep brain stimulation $\quad \bullet \quad$ pallidotomy

I

$\mathrm{N}$ the 1990s, publication of the long-term results of pallidotomy for advanced Parkinson's disease (PD) showing significant loss of dyskinesias, rigidity, and tremor led to a second resurgence of stereotactic surgery for PD. ${ }^{11}$ However, the relatively high incidence of side effects in some series and the demonstration that bilateral subthalamic nucleus (STN) stimulation was effective and led to a significant reduction in drug requirement, unlike pallidal surgery, resulted in the procedure of choice becoming deep brain stimulation (DBS). With the popularization of DBS, surgeon experience and confidence with stereotactic lesion-creating surgery has declined.

Infection of the implanted device poses a significant risk in DBS, more so for patients with limited mobility and ability to care for themselves. Furthermore, it is common for the population of patients with implanted DBS systems to survive into the late stages of PD, when implantable pulse generators continue to require replacement long after they have ceased to provide a benefit because of the natural history of the patient's cognitive decline. We propose that in such cases, a unilateral pallidotomy and removal of the DBS implant is socially and financially effective management. We also posit that pallidotomy may well have been abandoned too early.

\footnotetext{
Abbreviations used in this paper: DBS $=$ deep brain stimulation; $\mathrm{GPi}=$ globus pallidus internus; $\mathrm{PD}=$ Parkinson's disease; $\mathrm{STN}=$ subthalamic nucleus.
}

\begin{abstract}
Methods
For the years between 2008 and 2013, a retrospective chart review was conducted to collect data for patients who had undergone successful DBS to treat PD but who had their implants removed due to device infection or due to progression of cognitive decline. Four such cases were found and are described below.

The stereotactic technique employed by our service has been reported in detail previously. ${ }^{9}$ Neuroanatomical localization of the posteroventral globus pallidus internus (GPi) was performed using CT and MRI image fusion (Renishaw NeuroInspire). For pallidotomy, a Stereotactic TC Electrode (Cosman Medical Inc.) was passed to the target at the superior part of the optic tract in an awake patient. Using a $1.8-\mathrm{mm}$-diameter radiofrequency probe with a 2-mm exposed tip, macrostimulation was then used to confirm the position of the electrode with the production of visual phosphenes at $2 \mathrm{~V}$. The tip of the electrode was heated to $80^{\circ} \mathrm{C}$ for 30 seconds. The tip was then withdrawn $2 \mathrm{~mm}$ and heated for 60 seconds, and this was followed by a further 2-mm withdrawal and heating for 90 seconds. Dexamethasone was given at the start of the procedure and for 24 hours after the pallidotomy. Routine postoperative MRI was performed to assess the size and location of the lesion (Fig. 1).
\end{abstract}

\section{Case Studies}

Case 1

This 72-year-old man had idiopathic tremulous PD 


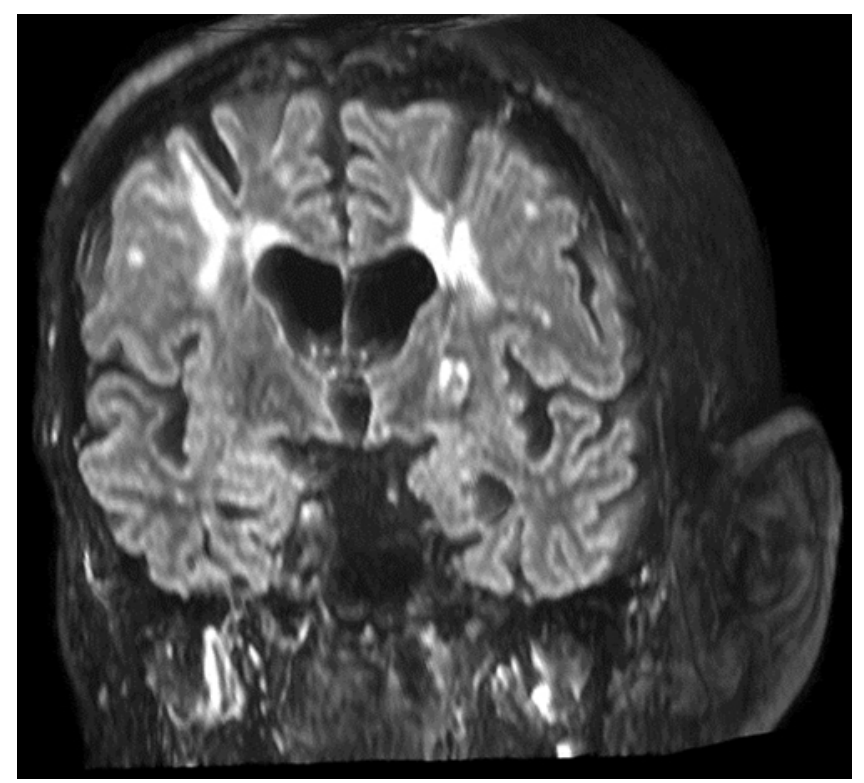

FIG. 1. Volumetric 3D T2-weighted MR image demonstrating the pallidotomy in the left GPi.

diagnosed when he was 58 years of age. In 2008, with a 10 -year history of motor complications (wearing-off, predictable-off, and dyskinesias), the patient was assessed for bilateral STN stimulation. In October 2008, he underwent subthalamic DBS and experienced motor benefit as a result. In February 2010, the left electrode was removed after it became infected, and failure to resolve the infection required removal of the remaining hardware 4 months later. Because of the motor fluctuations and dyskinesias, the patient underwent a right pallidotomy in October 2012. Consequently, his dyskinesias resolved and an improvement in his Unified Parkinson's Disease Rating Scale (UPDRS) score was maintained. Recently his UPDRS motor score was 19 and he has been taking Sinemet (25/250 mg every 3 hours), ropinirole ( $5 \mathrm{mg} 3$ times a day), and amantadine (2 times a day).

\section{Case 2}

This 63-year-old man was diagnosed with PD at the age of 49 years. He was assessed for bilateral STN stimulation 10 years later when he suffered severe dyskinesias and "off" freezing. In 2008, he underwent bilateral STN stimulation and had an excellent clinical result, but the system became infected and required removal. After appropriate antibiotic therapy, a second system was implanted a year later but again due to infection was removed. Because of these infections, an opportunity to undertake quantitative outcome scoring was not found. With no obvious cause for his repeated infections, he was offered a dominant (left-side) pallidotomy, which was performed without event and eliminated his dyskinesias.

\section{Case 3}

This 73-year-old patient with advanced PD, whose predominant problem was biphasic dystonia with severe drug-related dyskinesias, was diagnosed with the disease when he was 58 years of age. At the age of 65 years, he underwent implantation of bilateral pallidal stimulators with excellent symptom control. At follow-up examination 9 years after the original system was implanted, he was severely cognitively impaired, had hallucinations, and was unable to care for himself; he was taking warfarin for atrial fibrillation. Although the pulse generator had run out of energy, after a discussion the family elected for him to undergo a dominant (left-side) pallidotomy and removal of all the hardware. The procedure eliminated the patient's dyskinesias and dystonia, and his wife was then able to take him home without the burden of repeated future follow-up and pulse generator replacements. Cognitive impairment preventing reliable following of instructions precluded quantitative outcome scoring.

\section{Case 4}

This 70-year-old woman with PD was diagnosed at the age of 46 years; she had predominant left hand tremor. She developed severe dyskinesias and underwent implantation of bilateral pallidal stimulators at the age of 59 years. Eleven years after the implantation of the original device, the patient developed severe cognitive impairment and was unable to care for herself. A planned generator change was delayed because the patient had a concurrent urinary tract infection and methicillin-resistant Staphylococcus aureus (MRSA) colonization (she had a uterine prolapse that caused her to be vulnerable to recurrent urinary tract infections). Due to the high risk of device infection and to the patient's cognitive decline resulting from $\mathrm{PD}$ progression, it was elected to offer a dominant (leftside) pallidotomy and remove all the hardware. The procedure eliminated her dyskinesias and dystonia. Cognitive impairment preventing reliable following of instructions precluded quantitative outcome scoring.

\section{Discussion}

Since the late 1990s, DBS for PD has been widely used for the reduction of motor complications that are inadequately controlled by medications or for patients with medication-induced side effects. ${ }^{7,17}$ With the success of DBS, lesional surgery to alleviate motor symptoms has been infrequently performed. ${ }^{10}$ The undesirable behavioral effects of STN stimulation have limited the use of DBS to patients with little comorbidity and no cognitive or behavioral disorders. ${ }^{16}$ This restriction has resulted in a large number of patients with preexisting cognitive or behavioral symptoms or with end-stage PD not being considered for surgical intervention. Furthermore, in the coming years a large number of patients who have previously been successfully treated with DBS will experience disease progression, thus limiting the benefit obtained from stimulation. We propose that in such cases, patients should be considered for pallidotomy. However, it must be stressed that the pallidotomy should be considered a "salvage" procedure for 2 main reasons: 1) the reduction in motor complications is not as significant as that achieved with DBS, and 2) the alleviation of motor symptoms gain is asymmetrical, due to the inability to perform bilateral lesioning without significant side effects. 
An infection of the DBS hardware has a significant impact on the patient's quality of life and also represents a not inconsequential economic cost to the community. Treatment of such infections can range from long-term antibiotic regimens (with the aim of retaining a functioning system but with the risk of developing antibiotic-resistant organisms) to removal of various components of the device (with the consequence of abatement in control of motor fluctuations). Anecdotally, the generator appears to be the most common site of infection because repeated replacements are necessary due to a limited battery life. In rare cases, the entire system will need to be removed which will result in worsening PD symptoms. There appears to be a subgroup of patients who develop repeated infections after implantation despite hardware removal, antibiotic therapy, and repeat implantation. The reason for these patients' vulnerability is unclear but may be related to poor nutrition or high microbial skin load.

Globally, with over 100,000 patients estimated to have had DBS, the burden of long-term follow-up is likely to be significant in patients whose disease has advanced to a point where there is no significant benefit of DBS, due to disease progression and cognitive decline or repeated intractable infections. In the presence of severe cognitive decline, the decision to continue to replace pulse generators must be questioned, although clinically patients can benefit from the abolition of tremor, rigidity, and dyskinesias. All the patients described in the present series fall within this group. Only one patient did not have an infection; thus, the decision to proceed with a pallidotomy was based purely on our observation of the cognitive effects of progressive PD because stimulation had been previously successful in alleviating motor side effects. Due to the absence of stimulation-induced cognitive decline in this patient, we decided that removing a single lead or adopting GPi as a target would not improve the clinical benefit of stimulation. We believe that with the aging of our population of patients with implanted DBS devices, this clinical situation will become commonplace, and, rather than just ceasing stimulation due to progressive PD-related cognitive dementia, the patients could benefit from the creation of an appropriate lesion. We do not think that rechargeable pulse generators would represent a good costeffective strategy in this advanced group in comparison with pallidotomy lesions and, moreover, would confer the same infection risks and follow-up challenges seen with programming and DBS maintenance.

A unilateral pallidotomy has always been an effective procedure with, in experienced hands, a very acceptable risk profile. ${ }^{8,10}$ Its recent abandonment has resulted in a decline in the number of surgeons capable of performing it. This is unfortunate because a unilateral lesion improves tremor, rigidity, and dyskinesias and has demonstrated improvement shown up to 19 years after. ${ }^{3}$ There is minimal risk of worsening neuropsychological decline $\mathrm{e}^{1,15,18}$ and improvement in quality of life..$^{19}$ In the patients who require a pallidal lesion, it has been found that a unilateral lesion is not have associated with more postoperative complications than DBS, ${ }^{4}$ but the risk increases with a subsequent contralateral lesion. ${ }^{6}$ Our technique involved the use of radiofrequency ablation to cause thermocoagulation and, hence, the pallidotomy. We believe that the immediate ef- fect and the control over the lesion size make this superior to the Gamma Knife lesioning that has recently been described. ${ }^{12}$

Ablation can always be considered if DBS fails, but it may be appropriate as first-line surgical therapy when the efficacy of ablation is better established-for example, in psychiatric disorders. ${ }^{5}$ It is also of use in cases in which the disease is refractory to or unsuitable for DBSfor example, cingulotomy for obsessive compulsive head bangers who may damage their indwelling neuroprostheses, or for patients with cancer pain in whom the tolerance effect could continue up to a year and in whom the effect could occur beyond their limited lifespan. In cases in which DBS may be impractical due to cost and a patient's inability to attend follow-up such as that seen in rural, developing world settings, other lesioning procedures not widely performed like subthalamotomy for PD might be indicated. ${ }^{2}$

The status of lesioning in movement disorders was well summarized by Blomstedt and Hariz: "Unilateral ablative surgery may not harbour more postoperative complications or side effects than DBS. Some of the side effects following lesioning are transient and most but not all DBS side effects are reversible. In the Vim DBS is safer than lesioning, while in the pallidum, unilateral lesions are well tolerated."'

A quarter of a century of contemporary DBS experience has begun to clarify the procedure's limitations and opportunities for neurosurgical alternatives, as happened for the levodopa that heralded it. While reviews of lesioning exist, ${ }^{10,13}$ a systematic appraisal of the contemporary literature for deep brain ablation in the era of DBS is desirable. Psychiatric disorders, cancer pain, and selected cases of movement disorder remain appropriate indications. Furthermore, we predict a resurgence in ablative surgery driven by the encouraging renaissance of stereotactic and functional neurosurgery in resource-limited, developing world settings. ${ }^{14}$

\section{Conclusions}

To our knowledge, this is the first case series of PD patients who originally underwent DBS and then subsequently underwent implant removal and pallidotomy to control PD motor symptoms. In this group, the indications for pallidotomy were PD progression with subsequent cognitive decline, repeated postimplantation infections that required total removal of the stimulation hardware, and challenges in maintaining patient follow-up. When stopping stimulation, the consequent worsening of motor complications in this group has resulted in the patients being placed in high-level care. In each case, this situation was reversed by pallidotomy.

\section{Disclosure}

The authors report no conflict of interest concerning the materials or methods used in this study or the findings specified in this paper.

Dr. Bulluss was supported by the Stuart Morson Scholarship in Neurosurgery, Royal Australasian College of Surgeons. 


\section{K. J. Bulluss et al.}

Author contributions to the study and manuscript preparation include the following. Conception and design: all authors. Acquisition of data: Bulluss. Drafting the article: Bulluss, Pereira, Aziz. Critically revising the article: all authors. Reviewed submitted version of manuscript: all authors. Approved the final version of the manuscript on behalf of all authors: Bulluss. Study supervision: Aziz.

\section{References}

1. Alegret M, Valldeoriola F, Tolosa E, Vendrell P, Junqué C, Martínez J, et al: Cognitive effects of unilateral posteroventral pallidotomy: a 4-year follow-up study. Mov Disord 18:323328,2003

2. Alvarez L, Macias R, Lopez G, Alvarez E, Pavon N, Rodriguez-Oroz MC, et al: Bilateral subthalamotomy in Parkinson's disease: initial and long-term response. Brain 128:570-583, 2005

3. Blomstedt P, Hariz GM, Hariz MI: Pallidotomy versus pallidal stimulation. Parkinsonism Relat Disord 12:296-301, 2006

4. Blomstedt P, Hariz MI: Are complications less common in deep brain stimulation than in ablative procedures for movement disorders? Stereotact Funct Neurosurg 84:72-81, 2006

5. Cosgrove GR, Rauch SL: Stereotactic cingulotomy. Neurosurg Clin N Am 14:225-235, 2003

6. De Bie RM, Schuurman PR, Esselink RA, Bosch DA, Speelman JD: Bilateral pallidotomy in Parkinson's disease: a retrospective study. Mov Disord 17:533-538, 2002

7. Deuschl G, Schade-Brittinger C, Krack P, Volkmann J, Schäfer $\mathrm{H}$, Bötzel K, et al: A randomized trial of deep-brain stimulation for Parkinson's disease. N Engl J Med 355:896-908, 2006

8. Dewey RB Jr, Giller CA, Broline SK, Mendelsohn DB, Lacritz LH, Cullum CM: Clinical outcome of unilateral stereotactic pallidotomy without microelectrode recording for intractable Parkinson's disease. Parkinsonism Relat Disord 6:7-16, 2000

9. Green AL, Joint C, Sethi H, Bain P, Aziz TZ: Cost analysis of unilateral and bilateral pallidotomy for Parkinson's disease. J Clin Neurosci 11:829-834, 2004

10. Gross RE: What happened to posteroventral pallidotomy for Parkinson's disease and dystonia? Neurotherapeutics 5:281293, 2008

11. Laitinen LV, Bergenheim AT, Hariz MI: Leksell's postero- ventral pallidotomy in the treatment of Parkinson's disease. J Neurosurg 76:53-61, 1992

12. Ohye C, Higuchi Y, Shibazaki T, Hashimoto T, Koyama T, Hirai T, et al: Gamma knife thalamotomy for Parkinson disease and essential tremor: a prospective multicenter study. Neurosurgery 70:526-536, 2012

13. Okun MS, Vitek JL: Lesion therapy for Parkinson's disease and other movement disorders: update and controversies. Mov Disord 19:375-389, 2004

14. Pereira EA, Aziz TZ: Reincarnating the oxford cingulectomy in the epoch of stereotaxy and resurrecting lesions in the era of deep brain stimulation. Stereotact Funct Neurosurg 91:262263, 2013

15. Perrine K, Dogali M, Fazzini E, Sterio D, Kolodny E, Eidelberg D, et al: Cognitive functioning after pallidotomy for refractory Parkinson's disease. J Neurol Neurosurg Psychiatry 65:150-154, 1998

16. Rodriguez RL, Fernandez HH, Haq I, Okun MS: Pearls in patient selection for deep brain stimulation. Neurologist 13:253260, 2007

17. Williams A, Gill S, Varma T, Jenkinson C, Quinn N, Mitchell $\mathrm{R}$, et al: Deep brain stimulation plus best medical therapy versus best medical therapy alone for advanced Parkinson's disease (PD SURG trial): a randomised, open-label trial. Lancet Neurol 9:581-591, 2010

18. York MK, Lai EC, Jankovic J, Macias A, Atassi F, Levin HS, et al: Short and long-term motor and cognitive outcome of staged bilateral pallidotomy: a retrospective analysis. Acta Neurochir (Wien) 149:857-866, 2007

19. Zimmerman GJ, D'Antonio LL, Iacono RP: Health related quality of life in patients with Parkinson's disease two years following posteroventral pallidotomy. Acta Neurochir (Wien) 146:1293-1299, 2004

Manuscript submitted July 12, 2013.

Accepted August 22, 2013.

Please include this information when citing this paper: DOI: 10.3171/2013.8.FOCUS13293.

Address correspondence to: Kristian J. Bulluss, M.B.B.S., Ph.D., F.R.A.C.S., Department of Neurosurgery, Level 3, West Wing, John Radcliffe Hospital, Oxford OX3 9DU, United Kingdom. email: k_bulluss@hotmail.com. 